\title{
Prognóstico e complicações da estenose mitral na gestação: uma revisão narrativa
}

\author{
Prognosis and complications of mitral stenosis in pregnancy: a narrative review
}

Pronóstico y complicaciones de la estenosis mitral en el embarazo: una revisión narrativa

Laerte de Paiva Viana Filho"* , João Pedro Costa Apolinário', Joel Lima da Silva Neto², Lívia Maria Schneider Passos ${ }^{3}$, Maria Fernanda César Marson, Mariana Silva Alves ${ }^{5}$, Matheus Alves Pacheco ${ }^{6}$, Sibelly Vaz de Mello Loureiro Ferreira ${ }^{7}$.

\section{RESUMO}

Objetivo: Discorrer a respeito da estenose mitral (EM), com ênfase no prognóstico e nas complicações dessa cardiopatia na gravidez. Revisão bibliográfica: A EM reumática é a principal valvulopatia de risco para gestante, onde há o aumento a pressão atrial esquerda que, associada ao aumento da volemia durante a gestação, pode evoluir com congestão pulmonar e arritmia. Além desses riscos para a gestante, há maior risco de prematuridade e baixo peso ao nascer. No tratamento, deve-se realizar o controle da frequência cardíaca, de arritmias e o emprego de anticoagulantes. Em refratárias à terapia medicamentosa, a Valvuloplastia Mitral Percutânea por Cateter-balão (VMCB) está indicada, sendo a cirurgia aberta última opção de tratamento. Considerações finais: A morbidade da EM é cerca de $60 \%$, mas a mortalidade é de apenas $1 \%$. O prognóstico é favorável, pois as alterações da gestação regridem em até seis meses pós-parto. Ademais, o prognóstico está diretamente relacionado à classe funcional, grau de EM e presença de arritmias.

Palavras-chave: Complicações na gravidez; Estenose da valva mitral; Gravidez

\begin{abstract}
Objective: To talk about mitral stenosis (MS), with emphasis on the prognosis and complications of this heart disease in pregnancy. Bibliographic review: Rheumatic MS is the main risk valve for pregnant women, where there is an increase in left atrial pressure that, associated with an increase in blood volume during pregnancy, can progress with pulmonary congestion and arrhythmia. In addition to these risks for pregnant women, there is a greater risk of prematurity and low birth weight. In the treatment, heart rate control, arrhythmias and the use of anticoagulants should be performed. In refractory drugs, Percutaneous Mitral Balloon Valve (MVCB) is indicated, with open surgery being the last treatment option. Final considerations: The morbidity of MS is around $60 \%$, but the mortality is only $1 \%$. The prognosis is favorable, as pregnancy changes regress within six months postpartum. Furthermore, the prognosis is directly related to the functional class, degree of MS and the presence of arrhythmias.
\end{abstract}

Keywords: Pregnancy complications; Mitral valve stenosis; Pregnancy

\footnotetext{
1 Pontifícia Universidade Católica de Minas Gerais (PUC-MG), Betim - MG

*E-mail: laerteviana07@gmail.com

2 Universidade Federal do Recôncavo da Bahia (UFRB), Santo Antônio de Jesus - BA

${ }^{3}$ Faculdade de ciências médicas e da saúde de Juiz de Fora (FCMS JF), Juiz de Fora - MG

${ }^{4}$ Universidade Metropolitana de Santos (UNIMES), Santos - SP

${ }^{5}$ Universidade Federal Fluminense (UFF), Niterói - RJ

${ }^{6}$ Universidade Federal de Santa Catarina (UFSC), Florianópolis - SC

${ }^{7}$ Faculdade de Medicina de Barbacena (FAME-FUNJOBE), Barbacena - MG
}

SUBMETIDO EM: 5/2020

ACEITO EM: 6/2020

PUBLICADO EM: 8/2020 


\section{RESUMEN}

Objetivo: Hablar sobre la estenosis mitral (EM), con énfasis en el pronóstico y las complicaciones de esta enfermedad cardíaca en el embarazo. Revisión bibliográfica: La EM reumática es la válvula de riesgo principal para las mujeres embarazadas, donde hay un aumento en la presión auricular izquierda que, asociado con un aumento en el volumen de sangre durante el embarazo, puede progresar con congestión pulmonar y arritmia. Además de estos riesgos para las mujeres embarazadas, existe un mayor riesgo de prematuridad y bajo peso al nacer. En el tratamiento, se debe realizar el control de la frecuencia cardíaca, las arritmias y el uso de anticoagulantes. En medicamentos refractarios, está indicada la válvula de globo mitral percutánea (MVCB), siendo la cirugía abierta la última opción de tratamiento. Consideraciones finales: La morbilidad de la EM es de alrededor del $60 \%$, pero la mortalidad es solo del $1 \%$. El pronóstico es favorable, ya que los cambios en el embarazo regresan dentro de los seis meses posteriores al parto. Además, el pronóstico está directamente relacionado con la clase funcional, el grado de EM y la presencia de arritmias.

Palabras clave: Complicaciones del Embarazo; Estenosis de la Válvula Mitral; Embarazo

\section{INTRODUÇÃO}

A doença cardíaca é a principal causa não obstétrica de morte no ciclo gravídico puerperal sendo a valvopatia de etiologia reumática, principalmente a estenose mitral (EM), responsável por cerca de metade das cardiopatias nesse grupo (TARASOUTCHI F, et al., 2011). No período gestacional, há aumento de quarenta por cento do volume sanguíneo e da frequência cardíaca (FC) promovendo, então, aumento do gradiente pressórico sobre a válvula estenosada, o que evolui com o início ou o agravamento dos sintomas neste grupo populacional.

Além disso, a diminuição da resistência vascular sistêmica (RVS) e da pressão arterial (PA) associadas ao estado de hipercoagulabilidade aumentam o risco de fenômenos tromboembólicos (TARASOUTCHI F, et al., 2011; PERELSHTEIN O, et al., 2019). O prognóstico relaciona-se à classe funcional, antecedentes de insuficiência cardíaca e tromboembolismo, alterações eletrocardiográficas (fibrilação atrial e sobrecarga de câmaras cardíacas) e ecocardiográficas (disfunção ventricular, medida da área valvar e presença de hipertensão pulmonar) (TARASOUTCHI F, et al., 2011).

$\mathrm{Na} E \mathrm{EM}$, há resistência ao fluxo sanguíneo através da válvula em razão do espessamento e da imobilidade dos folhetos valvares sendo fundamentalmente uma sequela reumática (TARASOUTCHI F, et al., 2011). Outras possíveis causas descritas são as congênitas, secundárias às doenças infiltrativas, Lúpus Eritematoso Sistêmico (LES), Artrite Reumatóide (AR) e síndrome carcinóide (TARASOUTCHI F, et al., 2011; BONOW R, et al., 2008; CHANDRASHEKHAR Y, et al., 2009).

Frequentemente, há complicações durante a gravidez, que a partir das mudanças fisiológicas que ocorrem no organismo da mulher, torna-se mal tolerada evoluindo com deterioração clínica e com consequências tanto para a mãe, quanto para o concepto (UELAND K e METCALFE J, 1975). Gestantes com EM podem apresentar manifestações graves como insuficiência cardíaca e edema agudo de pulmão (BORN, 1996; FRANKEN, 1986).

O manejo da EM durante a gravidez continua sendo um desafio tanto para clínicos, quanto para obstetras e cardiologistas. É interessante salientar que, mesmo com os cuidados modernos e um sistema de saúde eficiente de alguns países, existem pacientes portadoras de EM que atingem a idade fértil sem serem diagnosticadas e manifestam a doença pela primeira vez na gravidez. Esse fato está mais associado a desfechos negativos. Além disso, pode haver uma deterioração clínica importante nesse período, independente do grau de estenose mitral basal (PERELSHTEIN O, et al., 2019; SURI V, et al., 2019).

A gestação acompanhada de uma alteração estrutural cardíaca representa uma condição importante e que deve receber atenção da assistência médica. Cardiopatias maternas correspondem a um dos principais componentes para a mortalidade materna em todo o mundo, como causa de mortalidade materna indireta, principalmente em países de baixa e média renda (WATKINS D, et al., 2012). 
O principal tratamento para pacientes sintomáticas consiste no emprego da terapia medicamentosa, com o uso de betabloqueadores e o repouso no leito, havendo a opção da Valvuloplastia Mitral Percutânea por Cateter-balão (VMCB) a ser considerada principalmente em pacientes com anatomia favorável. As medidas terapêuticas possuem alguns riscos que devem ser atentamente avaliados, como o risco de exposição do feto à terapia medicamentosa ou à radiação durante a VMCB (FRANKEN R, 1986; FIROUZI A, et al., 2019).

Um diagnóstico precoce e um bom acompanhamento pré-natal são fatores que podem contribuir com a evolução favorável do quadro, auxiliar na seleção de pacientes que necessitam de tratamentos e intervenções clínicas e cirúrgicas além de diminuir as complicações. Este estudo tem como objetivo discorrer sobre o prognóstico da EM durante a gravidez e identificar as complicações mais comuns decorrentes do quadro neste período.

\section{REVISÃO BIBLIOGRÁFICA}

No Brasil, a principal causa de EM é a febre reumática. Essa patologia está frequentemente associada a infecções, principalmente, as estreptocócicas. No entanto, em países desenvolvidos, a EM está mais relacionada à processos degenerativos e à calcificação relacionados ao processo de envelhecimento, ocorrendo, então, principalmente em pacientes com idade avançada. Outras causas menos frequentes, tanto em países desenvolvidos quanto em países subdesenvolvidos, mas que devem ser citadas, são as congênitas, as secundárias ao LES e a AR, a síndrome carcinoide, à Doença de Fabry, à lesão actínica pós radioterapia e ao uso de alguns medicamentos como os anorexígenos (TARASOUTCHI F, et al., 2017).

A febre reumática $(F R)$ aguda é uma das principais sequelas da infecção pelo estreptococo beta-hemolítico do grupo A de Lancefield, o também conhecido Streptococcus pyogenes, sendo esta bactéria a principal etiologia da EM em nosso meio. Sua incidência decresceu nas últimas cinco décadas no mundo ocidental, no entanto, em países subdesenvolvidos a EM reumática continua sendo a doença cardíaca valvar mais comum.

Em todo o mundo, estima-se que existam cerca de 15 milhões de casos de doença cardíaca reumática e cerca de quatrocentos e cinquenta mil novos casos de FR por ano. A FR leva à inflamação de todo o pericárdio (pancardite) e à inflamação da superfície valvar (valvulite) além provocar insuficiência mitral em seus estágios mais iniciais. Em países onde a FR é endêmica, a progressão da lesão mitral parece ser mais rápida, levando à EM grave em pacientes ainda jovens.

Os principais fatores de risco relacionados à pior progressão da lesão mitral são a gravidade da cardite, as recorrências da FR e o baixo nível socioeconômico da população afetada. Frequentemente, a manifestação clínica da EM ocorre somente quando há importante aumento do débito cardíaco. Isso pode acontecer de forma secundária à gestação, febre, arritmias, infecção, tireotoxicose ou até exercícios físicos de alta intensidade. A ocorrência e a deflagração de sintomas estão associadas ao mau prognóstico quando a EM permanece sem tratamento e sem acompanhamento médico.

A FR pode ser evitada quando as infecções agudas pelo estreptococo são diagnosticadas e tratadas com antibiótico de forma adequada e em tempo hábil. A principal infecção estreptocócica relacionada ao desenvolvimento da FR é a faringoamigdalite. Além disso, a profilaxia secundária se faz necessária visto que podem haver episódios de recorrência de cardite mesmo em pacientes que estejam assintomáticos. Essa deve ser feita por dez anos após o último episódio documentado de ataque ou até os vinte e um anos de idade para os pacientes que tiveram a forma leve ou a cardite curada. No entanto, em pacientes com acometimento grave da válvula que resulte em necessidade de troca valvar ou naqueles com evolução para a insuficiência cardíaca, a profilaxia secundária deve ser feita por toda a vida (WUNDERLICH N, et al., 2019).

Quanto à sua fisiopatologia, a EM é caracterizada por uma obstrução parcial ao influxo ventricular esquerdo secundário ao estreitamento do orifício valvar mitral (BANOVIC M e DACOSTA M, 2018). Tal mecanismo decorre do espessamento dos folhetos da válvula associado à calcificação e à fusão de comissuras. Ademais, associa-se a isso o encurtamento das cordoalhas tendíneas (TARASOUTCHI F, et al., 2011, SELZER A e COHN K, 1972). 
Quando se fala de forma específica da EM reumática, a lesão é caracterizada por espessamento e fusão das comissuras da valva mitral, promovendo o estreitamento de seu lúmen, fusão das cordoalhas tendíneas e dos músculos papilares subjacentes (WUNDERLICH N, et al., 2019). A elevação da pressão atrial esquerda transmite-se de forma retrógrada ao leito vascular do pulmão determinando a congestão pulmonar, edema do interstício, hipertensão pulmonar e desenvolvimento de sintomas de forma progressiva (TARASOUTCHI F, et al., 2011, CHANDRASHEKHAR Y, et al., 2009).

Durante o ciclo gravídico, ocorrem inúmeras alterações fisiológicas próprias deste período. Tais alterações são necessárias para atender ao aumento da demanda materna bem como para garantir circulação uteroplacentária adequada para o perfeito desenvolvimento fetal. O gatilho inicial é a redução da resistência periférica e, com isso, há redução da pressão arterial que promove ativação do sistema nervoso simpático e do sistema renina-angiotensina-aldosterona através da ativação dos barorreceptores.

Os fatores que contribuem para a redução da resistência periférica são a vasodilatação sistêmica, desenvolvimento de um novo circuito vascular uteroplacentário e retenção de fluidos com expansão em cerca de quarenta por cento do volume sanguíneo. Há aumento do débito cardíaco a partir do primeiro trimestre associado a alterações na contratilidade cardíaca relacionados às alterações hormonais próprias desse período (FU Q, 2018).

Além disso, há tendência à hipercoagulabilidade como mecanismo de prevenção aos sangramentos que podem ocorrer, no entanto, essa tendência promove aumento do risco para fenômenos tromboembólicos principalmente quando associado a cardiopatias de base (TARASOUTCHI F, et al., 2011; PERELSHTEIN O, et al., 2019). Há reversão de tais alterações em cerca de três a seis meses após o parto com o então retorno às condições basais iniciais (FU Q, 2018).

A EM é a doença valvar mais frequente no ciclo gravídico puerperal e a com maior chance de descompensação por conta das alterações hemodinâmicas características deste período (PERELSHTEIN O, et al., 2019; AVILA W, et al., 2003). Frequentemente, a primeira manifestação clínica da EM ocorre na gestação ou no puerpério imediato e, contrariando todas as expectativas, a deterioração clínica pode ocorrer independentemente da gravidade da EM basal (PERELSHTEIN O, et al., 2019). Dentre os sinais e sintomas, a paciente pode apresentar fadiga, dispneia, congestão pulmonar, arritmia paroxística supraventricular e fibrilação atrial sendo que o primeiro sintoma pode ainda ser um fenômeno tromboembólico (TEDOLDI C, et al., 2009).

O ecocardiograma (ECO) é o principal exame de imagem utilizado para avaliar a gravidade e as consequências hemodinâmicas da EM. Esse exame, permite uma avaliação detalhada da morfologia valvar, extensão do acometimento do aparelho mitral subvalvar e o possível envolvimento concomitante de outras valvas. Na maioria dos casos, o ECO transtorácico é suficiente para classificar a EM (WUNDERLICH N, et al., 2019). O ECO está indicado especificamente na gestação para avaliação da gravidade da lesão, das causas de mudanças de classe funcional e avaliação da discrepância entre a sintomatologia apresentada e a gravidade do dano valvar observado (TEDOLDI C, et al., 2009). O ECO transesofágico está indicado quando o primeiro exame é insuficiente para uma adequada avaliação e diante da necessidade de se excluir a presença de trombos intracavitários antes de um procedimento cirúrgico (WUNDERLICH N, et al., 2019).

A avaliação da gravidade da EM inclui a avaliação dos gradientes médios em repouso e o cálculo da área valvar. A lesão pode ser classificada como discreta quando a área é maior do que um centímetro e meio quadrado e possui gradiente médio em repouso menor do que cinco $\mathrm{mmHg}$; em moderada quando a área está entre um e um centímetro e meio quadrado e o gradiente pressórico entre cinco e dez $\mathrm{mmHg}$ e; por fim, em importante ou grave quando a área é menor do que um centímetro quadrado e o gradiente pressórico é maior do dez mmHg (Tabela 1) (TEDOLDI C, et al., 2009). 
Tabela 1 - Classificação ecocardiográfica da estenose mitral.

\begin{tabular}{lcc}
\hline Lesão (grau) & Área $\left(\mathbf{c m}^{\mathbf{2}}\right)$ & Gradiente médio em repouso $\mathbf{( m m H g )}$ \\
\hline Discreta & $>1,5$ & $<5$ \\
Moderada & 1,0 a 1,5 & 5 a 10 \\
Importante & $<1,0$ & $>10$ \\
\hline
\end{tabular}

Fonte: Filho LPV, et al., 2020. Baseado em dados Tedoldi C, et al., 2009.

A consagrada classificação funcional da New York Heart Association (NYHA) estratifica o grau de limitação imposto pela doença cardíaca. A classe funcional (CF) I é identificada quando o paciente não apresenta sintomas durante as atividades de seu cotidiano não havendo qualquer limitação à prática dessas; a CF II é caracterizada pela ocorrência de sintomas a partir de atividades cotidianas; na CF III os sintomas são desencadeados por atividades menos intensas do que as cotidianas ou ainda a partir de pequenos esforços e; por último mas não menos importante, a CF IV se caracteriza pela presença de sintomas mesmo em repouso. Nessa classificação o principal sintoma apresentado e valorizado é a ocorrência de dispneia (Tabela 2) (BOCCHI E, et al., 2009).

Tabela 2 - Classificação funcional NYHA.

\begin{tabular}{lc}
\hline Classe funcional I & $\begin{array}{c}\text { Ausência de sintomas (dispnéia) durante atividades cotidianas. A limitação para } \\
\text { esforços é semelhante à esperada em indivíduos normais. }\end{array}$ \\
\hline Classe funcional II & Sintomas desencadeados por atividades cotidianas. \\
\hline Classe funcional III & $\begin{array}{c}\text { Sintomas desencadeados em atividades menos intensas que as cotidianas ou } \\
\text { pequenos esforços. }\end{array}$ \\
\hline Classe funcional IV & Sintomas em repouso. \\
\hline
\end{tabular}

Fonte: Filho LPV, et al., 2020. Baseado em dados Bocchi E, et al., 2009.

Os riscos da gravidez são maiores e mais preocupantes em pacientes com classe funcional III, sobrecarga do ventrículo direito, FA e área valvar mitral menor do que um centímetro e meio quadrado. As complicações maternas mais frequentes são congestão pulmonar e arritmia supraventricular paroxística, seguida pelos fenômenos tromboembólicos (TARASOUTCHI F, et al., 2011). Pode ainda haver o desenvolvimento de FA, secundária ao aumento do tamanho do átrio esquerdo que predispõe à redução da velocidade de fluxo sanguíneo e à estase sanguínea. Com a progressão da doença, pode haver aumento da pressão na artéria pulmonar o que, com o tempo, leva a dilatação e hipertrofia do ventrículo direito evoluindo com insuficiência cardíaca direita em estágios mais avançados (WUNDERLICH N, et al., 2019).

Sessenta por cento das portadoras de EM moderada a importante evoluíram para CF III ou IV durante a gravidez, independentemente de serem previamente assintomáticas ou sintomáticas (TARASOUTCHI F, et al., 2011). Em gestantes, a sobrecarga de volume provoca aumento do gradiente através da válvula mitral, por isso, a área valvar deve ser o parâmetro utilizado para a estimativa acerca da gravidade da lesão (TEDOLDI C, et al., 2009). As consequências fetais mais frequentemente observadas de forma geral são a prematuridade em cerca de $16 \%$ dos casos e o baixo peso ao nascer em cerca de $19 \%$. Vale ressaltar que quanto maior o grau da lesão, maior é o risco tanto para a mãe quanto para concepto (PERELSHTEIN O, et al., 2019).

Comparando a EM grave e a EM leve, as mulheres com o quadro grave apresentam aumento significativo do risco de insuficiência cardíaca ( $68 \%$ em casos graves e $11 \%$ em casos leves), arritmias ( $33 \%$ em casos graves e $0 \%$ em casos leves) e hospitalizações ( $78 \%$ em casos graves e $11 \%$ em casos leves). O risco de resultados fetais adversos também é estratificado de acordo com a gravidade da EM havendo risco de parto prematuro $44 \%$ em casos graves e $5 \%$ em quadros leves), restrição de crescimento intrauterino fetal (33\% em casos graves e $16 \%$ em casos leves) e taxas de natimortos ( $11 \%$ em casos graves e $0 \%$ em casos leves) aumentam na EM grave (Tabela 3).

Desse modo, as mulheres com EM que planejam engravidar devem sempre ser encaminhadas para um centro de atendimento terciário com experiência em cardio-obstetrícia como forma de garantir um 
acompanhamento especializado para essas mulheres. Pacientes com EM moderada devem ser alertadas de que a gravidez é de alto risco e exigirá monitoramento cuidadoso e contínuo durante toda a gravidez (LAU E e DEFARIA Y, 2019). Vale ressaltar que em mulheres portadoras de esclerose múltipla um teste de esforço deve ser feito durante $o$ aconselhamento pré concepcional para avaliação da tolerabilidade da gravidez nesse grupo de pacientes (WUNDERLICH N, et al., 2019).

Tabela 3 - Riscos associados ao grau de estenose mitral em porcentagem.

\begin{tabular}{lll}
\hline & EM grave & EM leve \\
\hline Insuficiência cardíaca & $78 \%$ & $11 \%$ \\
\hline Arritmias & $33 \%$ & $0 \%$ \\
\hline Hospitalizações & $78 \%$ & $11 \%$ \\
\hline Parto prematuro & $44 \%$ & $5 \%$ \\
\hline Restrição do crescimento fetal & $33 \%$ & $16 \%$ \\
\hline Natimortos & $11 \%$ & $0 \%$ \\
\hline
\end{tabular}

Fonte: Filho LPV, et al., 2020. Baseado em dados: Lau E, et al., 2018.

A EM apresenta alta morbidade estimada em $66 \%$ em publicação brasileira (AVILA W, et al., 2003). No Registry Of Pregnancy and Cardiac Disease (ROPAC) de 1321 mulheres grávidas em todo o mundo entre 2007 e 2011, havia 13 mortes maternas sendo 4 atribuídas à EM (LAU E e DEFARIA Y, 2019; ROOSHESSELINK J, et al., 2013). Desse modo, observa-se que a mortalidade é baixa pelos bons resultados da Valvoplastia Mitral por Cateter Balão (VMCB) em mulheres com importante deterioração clínica a despeito da terapia medicamentosa adequada (TEDOLDI C, et al., 2009). Em referências internacionais, a mortalidade dessas pacientes é menor do que $1 \%$ sendo principalmente causada pela insuficiência cardíaca subjacente seguida das complicações tromboembólicas (PERELSHTEIN O, et al., 2019; WUNDERLICH N, et al., 2019).

O manejo da EM em gestantes continua sendo um desafio clínico tanto para o generalista, quanto para o obstetra e para o cardiologista. O gerenciamento desta patologia deve envolver a prevenção da recorrência da FR, a melhora dos sintomas e a redução dos eventos tromboembólicos associados (WUNDERLICH N, et al., 2019). Desse modo, o tratamento envolve o controle da frequência cardíaca, controle de arritmias e a anticoagulação (LAU E e DEFARIA Y, 2019).

Em gestantes sintomáticas, indica-se a restrição da atividade física e controle da frequência cardíaca com o uso de betabloqueadores ou de bloqueadores dos canais de cálcio, naquelas com contraindicações ao uso do primeiro grupo de medicamentos (TEDOLDI C, et al., 2009). No entanto, alguns pesquisadores sugerem que o uso de betabloqueadores na gestação contribui com o desenvolvimento de restrição do crescimento fetal, mas, na ausência de ensaios clínicos controlados e randomizados acerca do tema, seu uso permanece controverso (LAU E e DEFARIA Y, 2019; ERSBOLL A, et al., 2014; TANAKA K, et al., 2016).

Caso a paciente apresente sinais de congestão, o uso de diuréticos como a furosemida está indicado. Se houver insuficiência cardíaca direita ou FA associadas, os digitálicos estão indicados. Em gestantes com EM complicada com fibrilação atrial e instabilidade hemodinâmica a cardioversão elétrica é eficaz. Caso haja necessidade de uma droga antiarrítmica para manter o ritmo sinusal, devido à intolerância aos sintomas, a quinidina e a procainamida são os medicamentos de escolha nesse cenário. Os betabloqueadores são preferíveis para controle da frequência ventricular, os bloqueadores de canal de cálcio (verapamil ou diltiazem) geram mais hipotensão materna, especialmente se administrados por via endovenosa (BONOW R, et al., 2008; WILSON W, et al., 2008).

A anticoagulação deve ser continuada na gestação. A heparina não fracionada (HNF) e a heparina de baixo peso molecular (HBPM) não atravessam a barreira placentária e, por isso, são os medicamentos preferíveis durante a gestação. No entanto, seu uso prolongado está associado ao estabelecimento de efeitos colaterais maternos tais como a trombocitopenia, hemorragia e osteoporose aliado ao desconforto para aplicação, dificuldade no controle da coagulação e o custo. A HBPM apresenta maior biodisponibilidade e maior meia vida quando comparada à HNF além de causar menos trombocitopenia. 
Contudo, tem maior custo (HIRSH J e RASCHKE R, 2004). O warfarin, antagonista da vitamina K, deve ser evitado no primeiro trimestre por sua capacidade de atravessar a barreira placentária causando efeitos teratogênicos conhecidos, especialmente entre a sexta e a nona semana. As malformações congênitas ocorrem em 1 a 3\% dos casos e o aborto espontâneo em 10 a 30\% dos casos.

Ademais, esse medicamento pode ainda acarretar restrição do crescimento intrauterino, prematuridade, anomalia do sistema nervoso central e morte neonatal secundária à hemorragia cerebral por compressão do polo cefálico no período expulsivo do parto (COTRUFO M, et al., 2002; ÁVILA W, 2010). Naquelas gestantes com insuficiência cardíaca severa e que não respondem à terapia medicamentosa, a intervenção valvar está indicada sendo a VMCB a intervenção preferível em gestantes com EM grave e anatomia favorável. A taxa de sucesso deste procedimento é próxima à cem por cento (LAU E e DEFARIA Y, 2019).

Contudo, deve ser evitada no primeiro trimestre para evitar exposição fetal à radiação (TEDOLDI C, et al., 2009). A adequada indicação da VMCB depende da mobilidade, da flexibilidade do folheto valvar e da extensão subvalvar do processo de fibrose. Tais parâmetros são avaliados por diversas classificações e sistemas que levam em conta achados ecocardiográficos, ressaltando a importância da realização do exame.

As principais contraindicações à VMCB são a presença de trombo em câmara cardíaca esquerda, EM leve, calcificação grave ou bicomissural, ausência de fusão comissural, doença valvar concomitante grave e doença arterial concomitante que requeira cirurgia (WUNDERLICH N, et al., 2019).

As complicações relacionadas à VMCB são raras, mas a paciente pode evoluir com tamponamento cardíaco, hemorragia, piora da EM, FA e até mesmo embolização sistêmica além da possibilidade comprometimento fetal secundário à exposição à radiação (LAU E e DEFARIA Y, 2019).

A substituição cirúrgica da válvula fica reservada para os casos mais graves que não tem indicação para a VMCB (LAU E e DEFARIA Y, 2019). Em estudo realizado fora do Brasil, com um grupo de quarenta e cinco mulheres com EM grave, a mortalidade fetal no grupo submetido a VMCB foi próxima a cinco por cento em comparação com trinta e oito por cento no grupo submetido a cirurgia aberta. Ademais, houve uma morte materna no grupo submetido a VMCB em contraste com oito óbitos maternos no grupo submetido à cirurgia (LAU E e DEFARIA Y, 2019; DE SOUZA J, et al., 2001).

Em relação à monitorização hemodinâmica durante o trabalho de parto, em mulheres com EM severa ou sinais e sintomas de insuficiência cardíaca grave no momento do parto, a monitorização invasiva por cateter no coração direito é apropriadamente indicada. Os fluidos devem ser manejados com o objetivo de alcançar uma pressão capilar pulmonar de quatorze $\mathrm{mmHg}$, embora esta possa ser ajustada com base na individualização de cada caso. A monitorização invasiva deve ser mantida no período de pós-parto imediato, devido aos grandes deslocamentos intravenosos de volume. A cesariana deverá ser realizada somente por indicação obstétrica (WILSON W, et al., 2008).

No que tange à profilaxia com antibiótico para endocardite, ela não se faz necessária para o parto cesariano e, muito menos, para o vaginal. Entretanto, a continuação dos antibióticos para a profilaxia secundária da FR deve ser indicada (BONOW R, et al., 2008).

\section{CONSIDERAÇÕES FINAIS}

A EM é a valvulopatia mais frequente na população brasileira sendo ainda a com maior risco de descompensação durante o ciclo gravídico puerperal. O aumento da pressão atrial esquerda se transmite determinando congestão pulmonar e arritmia supraventricular paroxística, sendo essas as complicações mais frequentemente presentes em gestantes. Dentre as complicações fetais, podemos citar a prematuridade e o baixo peso ao nascer como as principais. A morbidade estimada é de $63,5 \%$, mas com mortalidade inferior à 1\%. O tratamento envolve o controle da frequência cardíaca, controle de arritmias e anticoagulação. Nas que não respondem ao tratamento medicamentoso, VMCB está indicada. O prognóstico materno está diretamente relacionado à classe funcional, grau de EM e presença de arritmias; contudo, é favorável, visto que há reversão das alterações fisiológicas da gestação em até 6 meses após o parto com, então, retorno às condições basais iniciais. 


\section{REFERÊNCIAS}

1. AVILA WS, et al. Pregnancy in patients with heart disease: experience with 1000 cases. Clin Cardiol, 2003; 26: 13542.

2. AVILA WS. Anticoagulation during pregnancy of woman with heart diseases. J Vasc Bras, 2010; 9(1): 42-45.

3. BANOVIC M, DACOSTA M. Degenerative mitral stenosis: from pathophysiology to challenging interventional treatment. Curr Probl Cardiol, 2018; 44: 10-35.

4. BOCCHI EA, et al. III Diretriz Brasileira de Insuficiência Cardíaca Crônica. Arq Bras Cardiol, 2009; 93(1): 1-71.

5. BONOW RO, et al. Focused update incorporated into the ACC/AHA 2006 guidelines for the management of patients with valvular heart disease: a report of the American College of Cardiology/American Heart Association Task Force on Practice Guidelines (Writing Committee to Revise the 1998 Guidelines for the Management of Patients With Valvular Heart Disease): endorsed by the Society of Cardiovascular Anesthesiologists, Society for Cardiovascular Angiography and Interventions, and Society of Thoracic Surgeons. Circulation,2008; 118(15): 523-661.

6. BORN D. Insuficiência e estenose mitral na gravidez: análise de variáveis maternas e do concepto. Tese de Doutorado - Escola Paulista de Medicina - Universidade Federal de São Paulo,1996.

7. CHANDRASHEKHAR Y, et al. Mitral stenosis. Lancet, 2009; 374(9697): 1271-83.

8. COTRUFO M, et al. Risk of warfarin during pregnancy with mechanical valve prostheses. Obstet Gynecol, 2002; 99 : 35-40.

9. DE SOUZA JA, et al. Percutaneous balloon mitral valvuloplasty in comparison with open mitral valve commissurotomy for mitral stenosis during pregnancy. Journal of the American College of Cardiology, 2001; 37(3): 900-3.

10. ERSBOLL AS, et al. Treatment with oral betablockers during pregnancy complicated by maternal heart disease increases the risk of fetal growth restriction. International journal of obstetrics and gynaecology, 2014; $121(5)$ : 618 26.

11. FIROUZI A, et al. Percutaneous Transluminal Mitral Commissurotomy in Pregnant Women with Severe Mitral Stenosis. J Tehran Heart Cent, 2019; 14(1): 12-17.

12. FRANKEN RA. Edema agudo de pulmão na gravidez. Arq Bras Cardiol,1986; 47: 413-5.

13. FU Q. Hemodynamic and Eletrocardiographic Aspects on Uncomplicated Singleton Pregnancy. Adv Exp Med Biol, 2018; 1065: $413-431$.

14. HIRSH J, RASCHKE R. Heparin and low-molecular-weight heparin: The Seventh ACCP Conference on Antithrombotic and Thrombolytic Therapy. Chest, 2004; 126(3): 188-203.

15. LAU E, DEFARIA Y. Management of High Rick Cardiac Conditions in Pregnancy: Anticoagulation, Severe Stenotic Valvular Disease and Cardiomyopathy. Trends Cardiovasc Med, 2019; 29(3): 162-163.

16. PERELSHTEIN O, et al. Maternal and Neonatal Complications of pregnant Women with Mitral Stenosis. Isr Med Assoc J, 2019; 21(2): 88-93.

17. ROOS-HESSELINK JW, et al. Outcome of pregnancy in patients with structural or ischaemic heart disease: results of a registry of the European Society of Cardiology. European heart journal, 2013; 34(9): 657-65.

18. SELZER A, COHN KE. Natural history of mitral stenosis: a review. Circulation, 1972; 45(4): 878-90.

19. SURI V, et al. Factors affecting the outcome of pregnancy with rheumatic heart disease: an experience from lowmiddle income country, Journal of Obstetrics and Gynaecology, 2019; 39(8): 1087-1092.

20. TANAKA K, et al. Beta-Blockers and Fetal Growth Restriction in Pregnant Women With Cardiovascular Disease. Circulation journal. Official journal of the Japanese Circulation Society, 2016; 80(10): 2221-6.

21. TARASOUTCHI F, et al. Diretriz Brasileira de Valvopatias - SBC 2011 / I Diretriz Interamericana de Valvopatias SIAC 2011. Arq Bras Cardiol,2011; 97(5): 1-67.

22. TARASOUTCHI F, et al. Atualização das Diretrizes Brasileiras de Valvopatias: Abordagem das Lesões Anatomicamente Importantes. Arq Bras Cardiol,2017; 109(6): 1-34.

23. TEDOLDI CL, et al. Diretriz da Sociedade Brasileira de Cardiologia para Gravidez na Mulher Portadora de Cardiopatia. Arq Bras Cardiol,2009; 93(6): 110-178.

24. UELAND K, METCALFE J. Circulatory changes in pregnancy. Clin Obstet Gynecol,1975; 18: 41-60.

25. WATKINS DA, et al. The burden of antenatal heart disease in South Africa: a systematic review. BMC Cardiovasc Disord, 2012; 12: 23.

26. WILSON W, et al. Prevention of Infective Endocarditis - Guidelines From the American Heart Association: A Guideline From the American Heart Association Rheumatic Fever, Endocarditis, and Kawasaki Disease Committee, Council on Cardiovascular Disease in the Young, and the Council on Clinical Cardiology, Council on Cardiovascular Surgery and Anesthesia, and the Quality of Care and Outcomes Research Interdisciplinary Working Group. J Am Dent Assoc, 2007; 116(15): 1736-54.

27. WUNDERLICH NC, et al. Rheumatic Mitral Valve Stenosis: Diagnosis and Treatment Options. Curr Cardiol Rep,2019; 21(3): 14. 